\title{
An Urban Forest-Inventory-and-Analysis Investigation in Oregon and
}

\section{Washington}

\author{
Jacob L Strunk ${ }^{1}$, John R Mills ${ }^{2}$, Paul Ries $^{3}$, Hailemariam Temesgen ${ }^{4}$, Lacey Jeroue ${ }^{5}$
}

\footnotetext{
$1 *$ Corresponding author. Dept. of Forest Engineering, Resources and Management, Peavy 204, Oregon State University, Corvallis, Oregon 97331-5706, USA, Ph: (360)-902-1653, Fax: (541)-7374316, Jacob.Strunk@dnr.wa.gov
}

${ }^{2}$ USDA Forest Service Pacific Northwest Research Station, University of Washington, P.O. Box 3890, Portland, OR 97208-3890, USA, JMills@,fs.fed.us

${ }^{3}$ Dept. of Forest Ecosystems and Society, Oregon State University, Corvallis, 321 Richardson Hall, Oregon 97331-5706, USA, Paul.Ries@,oregonstate.edu

${ }^{4}$ Dept. of Forest Engineering, Resources and Management, Oregon State University, Corvallis, Peavy 204, Oregon 97331-5706, USA, Hailemariam.Temesgen@oregonstated.edu

${ }^{5}$ Dept. of Forest Engineering, Resources and Management, Oregon State University, Corvallis, Peavy 204, Oregon 97331-5706, USA, ljeroue@west-inc.com 


\section{Abstract}

2 The U.S. Department of Agriculture (USDA) Forest Service, Forest Inventory and Analysis

3 program recently inventoried trees on 257 sample plots in the urbanized areas of Oregon and

4 Washington. Plots were located on the standard grid $(\approx 1$ plot / 2428 ha $)$ and installed with the 4-

5 subplot footprint ( $\approx .067$ ha with 4 circular subplots). Using these data, we examined: 1$)$ use of

6 the land use classification data from the National Land Cover Database (NLCD) for post-

7 stratification; 2) the resolution of the inventory data to make inferences about subdomains

8 (specifically sub-regions) and subgroups (species and diameter classes); and 3) the i-Tree Eco

9 software as a tool for data compilation, estimation, and reporting.

10 Ideally post-stratification would enable us to achieve greater precision in sub-regions (Seattle,

11 Portland, and eastern Oregon and Washington), but our analyses indicated that NLCD land use

12 classes did not aid us in estimation of trees per ha and basal area in Oregon and Washington

13 urbanized areas. Due to the small sample size and lack of effective post-stratifying variables, the

14 data support few inferences about sub-regions. It is likely, however, that another set of stratifying

15 variables can improve precision and enable a greater diversity of sub-region inferences from

16 these data. 
18 Urban forests provide a myriad of environmental, social, and economic benefits for what the US

19 Census estimates is approximately 80 percent of the U.S. population. The term "urban forest" is

20 used to define all trees within an urban area, including those along parking strips, in yards, and

21 on public lands such as parks (Cumming et al., 2008). It is a mosaic of both the planted

22 landscape and native forest remnants that have been left behind, intentionally or unintentionally,

23 as our cities have developed. Even vacant or previously cleared land, when left long enough, can

24 revert back to forest by natural processes. Research conducted in the last several decades has

25 begun to quantify many of thebenefits provided by urban trees, which include reduced storm

26 water runoff (Sanders, 1986), cleaner air (Nowak, 1994; Nowak et al., 2006), support for better

27 mental and physical health (Ulrich et al., 1991; Dwyer et al., 1991; Donovan et al., 2013; Kardan

28 et al., 2015), reduced crime (Kuo and Sullivan, 2001; Donovan and Prestemon, 2012), reduced

29 home energy costs (Akbari, 2002; Donovan and Butry, 2009), and trees make neighborhoods

30 more desirable places to live and work (Westphal, 2003; Wolf, 2003; Kinzig et al., 2005; Nowak

31 and Dwyer, 2007). The Arbor Day Foundation reports that more than $\$ 1$ billion is spent on

32 planting, maintaining, and managing the urban forest in the over 3,400 cities awarded Tree City

33 USA status (“Arbor Day Foundation," n.d.). Given the importance of urban forests, and the large

34 amounts of public funds spent to manage them, very little is actually known about the health,

35 extent, and characteristics of the nation's urban forest resource. To help remedy this situation,

36 the Forest Inventory and Analysis (FIA) program of the USDA Forest Service has recently begun 
37 several projects to collect data in urban areas for the purpose of preforming analyses and

38 informing the public on the status and trends of urban forests.

39 The FIA program originated in 1930 as an effort to "make and keep current a comprehensive

40 inventory and analysis of the present and prospective conditions of, and requirements for, the

41 renewable resources of the forest and rangelands of the U.S." ( U.S. Department of Agriculture

42 Forest Service, 1992). Every year, in every U.S. state, the FIA program collects data from a set

43 of permanent ground plots, then analyzes and later reports information on the extent and health

44 of states' forest resources. While the FIA plot sample grid covers all the land area in each state,

45 for a plot on the grid to be installed it had to intersect a forested area, defined as at least 0.4 ha in

46 size, at least $36.5 \mathrm{~m}$ wide, and at least 10 percent stocked with tree canopy and containing an

47 understory that is undisturbed by another land use (U.S. Department of Agriculture Forest

48 Service, 2012). The plots located outside of these conditions have been considered nonforest or

49 developed, and they were not installed. In the last decade, an effort has begun to include urban

50 land in the sample. This is based on the understanding that across the landscape there is a gap in

51 the ability to account for trees and their potential benefits outside of the traditional forests. As

52 part of an all-lands approach, FIA acknowledged the importance of urban trees and they have

53 focused recent efforts on installing plots located in several urban areas.

54 Many cities rely on ground based tree inventories of street and park trees, or on aerial based

55 canopy coverage studies to obtain data for planning and management purposes. Effective forest

56 inventory design, analyses, and reporting typically require a suite of specialized skills that may

57 not be available within an organization. Some cities look to contractors and volunteers to assist 
58 in inventory collection, and it is common for cities to perform minimal inventories that are on

59 decade intervals and on limited land use types. This can prove problematic for data continuity if

60 different contractors or measurement protocols are used between inventories. Additionally, since

61 the inventories are not standardized among cities, they may prove useless for policy and planning

62 at the state, regional or national level (Cumming et al., 2008).

63 The difficulties described in implementing a forest inventory suggest that there is a need for

64 infrastructure to support consistent urban inventory practices, as well as to provide inventory

65 analyses and to communicate inventory results. Software packages that provide inventory

66 guidance, analyses, and reporting capacity may help alleviate some of the difficulties associated

67 with urban inventory and reduce the overhead associated with planning and maintaining an

68 inventory system. In addition, if municipalities across the region adopted a standard

69 methodology, reporting and planning may be feasible at a larger scale.

70 The first FIA inventory of nonforest conditions in urban areas was in 1999, when FIA initiated

71 an assessment of all non-forest plots in the 5 counties surrounding the city of Baltimore,

72 including both urban and rural lands (Riemann, 2003). In 2001, the USDA Forest Service,

73 Forest Health and Monitoring (FHM) program initiated an assessment of urban forest conditions

74 (Cumming et al., 2001). This assessment delimited urban boundaries and then collected tree

75 information from established plots within the urban boundaries. This was the first attempt to

76 apply national forest health monitoring protocols to aid in the planning and management of the

77 urban forest. Statewide urban pilot studies were later conducted by FIA incorporating the

78 protocols developed by the FHM program. More recently, urban areas in five states were 
79 inventoried by FIA including Indiana, Wisconsin, Colorado, New Jersey, and Tennessee (Nowak

80 et al., 2011; Cumming, 2007; Lake et al., 2006). Data obtained from plots can be used to monitor

81 the health, condition, and trends of the urban forest, giving managers an important tool for long

82 range planning.

83 In 2009, with funding from the America Recovery and Reinvestment Act of 2009, the USDA

84 Forest Service Pacific Northwest Research Station (PNW) and the Oregon Department of

85 Forestry (ODF) entered into an agreement to conduct an FIA inventory project in the "urbanized

86 areas" of five Pacific coast states (Alaska, California, Hawaii, Oregon, and Washington).

87 Urbanized areas are defined by U.S. census to contain a core population of 50,000 people. This

88 classification was chosen because 88 percent of the U.S. urban population lived in urbanized

89 areas in 2010 and funding was not sufficient to install plots in the less densely populated land

90 classification "urban clusters" (for classification information see:

91 https://www.census.gov/geo/reference/ua/urban-rural-2010.html). In coordinating with the PNW

92 FIA Program, ODF appointed a project manager, project coordinator, and quality assurance

93 forester to help manage the project, and hired private urban forestry and forest inventory firms to

94 collect the data. PNW formed an agreement with California Polytechnic State University, San

95 Luis Obispo to collect the California urban data. The Hawaii and Alaska data will be published

96 by the PNW FIA, but their relatively small urban areas limit analyses.

97 The objective of this study is to investigate our ability to make inferences about the character of

98 the urban forest from urban FIA field data collected in 2010 and 2011 for Oregon and

99 Washington. Initially we investigate whether NLCD data can be used to improve the precision of 
100 our estimates. We then investigate whether the collected inventory data support estimation

101 within sub-regions and for subgroups. Finally, we examine i-Tree Eco (Nowak et al., 2013)

102 software as a tool for estimation and reporting of selected base inventory estimates.

103 Methods

104 PNW urban inventory overview

105 Plots on the national FIA grid are spaced so each represents roughly 2428 ha. According to GIS

106 layers provided by the U.S. Department of Commerce, Bureau of the Census (2011), there are

107 163K ha of urbanized land in Oregon state and 460K ha in Washington state. A total of 67 plots

108 from the FIA grid fall within Oregon's urbanized areas and 190 fall within Washington's

109 urbanized areas. Plot locations were mapped using coordinates derived from maps, aerial photos,

110 and satellite imagery. Landowners were determined using GIS layers available from public

111 agencies. All the plots in urban areas are part of the national FIA plot grid, and some plots on the

112 edges of urban areas were already installed because they fell in area that met FIA's definition of

113 forest. The majority of urban plots, however, were previously classified as nonforest and had not

114 been installed. Forested FIA plots in the PNW region are measured on a panel system in which

115 one-tenth of all the plots within a State are measured in a given year. Because this study was

116 funded with a one-time grant, all plots in the urban areas were measured as urban plots in a two

117 year window. 
119 Field data were collected in the summer of 2010 and 2011. The standard FIA plot design was 120 used, in which each plot consisted of four subplots, each 0.017 ha in size with a nested microplot 121 covering 0.0013 ha (Fig. 1).

Figure 1. FIA plot design

123 Urban protocols were based on a supplement to the standard FIA field manual that included a

124 subset of tree health variables from Forest Health plots (Tallent-Halsell, 1994), and variables

125 unique to the urban environment (e.g., poor pruning, conflicts with tree roots, etc.) (U.S.

126 Department of Agriculture Forest Service, 2012). The spatial locations of plots were obtained in 127 the field using GPS units - which became the official plot coordinates. All trees with a diameter 128 at breast height $(\mathrm{DBH})$ of $12.7 \mathrm{~cm}$ and greater were mapped and measured on each subplot and 129 trees from $2.54 \mathrm{~cm}$ to $12.7 \mathrm{~cm}$ were measured and mapped on each microplot. Tree variables 130 included: species, diameter, height, and several variables to assess tree health based on crown 131 attributes, variables to account for tree damage, the presence or absence of pests, and the distance

132 of tree to nearby buildings. Subplot variables include the percent of canopy, shrub, and ground 133 cover on each subplot, the surface composition (impervious or permeable), and land uses. A 134 summary of some basic measurements performed on field plots is provided in Table 1.

135

Table 1

137 Field data were recorded using a personal digital assistant (PDA) running software specifically 138 designed by the USDA Forest Service for the collection of urban FIA data. This software, 
139 known as Midas, was implemented nationally with versions for both forest and urban data

140 collection.

$141 \quad$ Post-Stratification with NLCD classes

142 An important strategy to improve our capacity to make inferences from samples is to leverage

143 auxiliary sources of information when performing estimation. Stratification is a common method

144 to improve estimation precision in which plots are associated with homogeneous groups

145 according to auxiliary information. This process may be performed before the inventory (pre-

146 stratification, or simply stratification) or after the inventory is completed (post-stratification).

147 One source of auxiliary information that has been used with favorable results with FIA data is

148 the National Land Cover Database (NLCD). NLCD is a $30 \mathrm{~m}$ resolution raster dataset which uses

149 Landsat data (30 m pixels) and unsupervised classification to predict land cover classes. The

150 Landsat-based resource has the advantage of being free and available over the entire contiguous

151 U.S. In inventories of forested areas this has worked well. Dunham et al. (2001), for example,

152 obtained a $15 \%$ improvement in their standard error for the mean volume estimate by post-

153 stratifying on NLCD classes (relative to estimation without stratification). The study by Liknes

154 et al. (2006) observed slightly more dramatic improvements (21\% to 68\%) in precision by post-

155 stratifying on NLCD for numbers of trees, biomass, and volume. Liknes et al. (2006) saw even

156 slightly better post-stratification results by using data from the U.S. Department of Agriculture

157 cropland data layer (http://www.nass.usda.gov/research/Cropland/SARS1a.htm), another remote

158 sensing based classification system. 
159 In this study we investigated our ability to post-stratify our sample into more homogeneous

160 groups for our region of interest - urbanized areas in Oregon and Washington - based upon land

161 cover classification. The data were grouped using 2006 version NLCD classes (Fry et al., 2011).

162 The NLCD classification system was evaluated for its level of agreement with field data, as well

163 as for its contribution to estimation. We used the level of association between NLCD and field-

164 measured land use classes as a quasi-measure of accuracy. Four indices of association were used:

165 Cramer's V (Cramér, 1946), Goodman-Kruskal lambda and tau (Goodman and Kruskal, 1954),

166 and Theil's uncertainty coefficient (Theil, 1967). These statistics were computed using the

167 StatMatch package (D'Orazio, 2012) in R (R Development Core Team, 2010). In a second

168 strategy we evaluated land use classes by looking at the separation in a measured forest attribute

169 between groups following grouping observations by land uses classes. Lastly, we compared

170 standard errors with and without post-stratification.

171 
173 Subdomains (sub-regions) and subgroups

174 Estimates were calculated for all urbanized areas in Oregon and Washington combined and for

175 subdomains (sub-regions) Oregon, Washington, Portland in Oregon, Seattle in Washington, and 176 urban areas in eastern Oregon and Washington (Fig. 2) where a subdomain is a partition of the 177 target population. We estimated the number of trees per hectare (TPH) and square meters per 178 hectare of tree basal area (BA) for all of the data combined and for the sub-regions by species 179 and $5 \mathrm{~cm}$ diameter classes (DBH, diameter at breast height). Our evaluation of estimation in sub180 regions and subgroups was based upon associated confidence intervals and t-statistics. For the 181 indicated sub-regions and subgroups we were interested in whether there were sufficient 182 numbers of observations to support inferences about TPH and BA. This analysis is meant to 183 highlight the resolution of these data for making inferences about subcomponents of the 184 population.

185 i-Tree Eco Software

186 We examined i-Tree Eco software as a tool for compilation and estimation of urban inventory 187 data. Estimation of base variables with i-Tree Eco was compared with estimation performed in 188 R. We did not attempt to validate i-Tree Eco model-based predictions of tree attributes such as 189 biomass, carbon, or any of the other environmental attributes. Our examination of this software 190 was chiefly aimed at feasibility and function for estimation of primary forest inventory attributes 191 - e.g., were we able to integrate our urban FIA measurements with this free software and achieve 192 reasonable estimates of TPH by species and size with associated errors. 
193 A strength of the i-Tree Eco software is that it is free and easy to use (when data are collected

194 with their software) and it is accompanied by a standardized measurement protocol. While the

195 software is developed with the support of USDA Forest Service research, the software is not

196 designed to accept field measurements directly from FIA plots. The i-Tree software was

197 designed to utilize more intensive city inventories and output a broad suite of results at the city

198 scale, rather than accept FIA field measurements from larger areas. Though the data collected is

199 similar, one of the hurdles that may arise for organizations planning to use the software in

200 conjunction with an FIA inventory is formatting the data for use with the complex structure of

201 the i-Tree Eco database. This includes handling FIA's multiple subplots, measurements from

202 subplots with different unit areas, and accepting data from multiple cities.

Figure 2. Urban areas in OR and WA by sub-regions.

205 To evaluate i-Tree Eco with urban FIA data, the FIA data were reformatted in R and deposited

206 into the appropriate tables in the i-Tree Eco. Our ad hoc solution used the RODBC package

207 (Ripley, 2012) in R to interact with the i-Tree Eco database. Linking external data with i-Tree

208 Eco required first parsing all of the spreadsheets to identify linked columns, and requires that the 209 user parse each field and identify the data types of each record.

\section{Results}

211 The results are presented in three sections. In the first section we evaluate NLCD land classes

212 and examine their contribution to improve the post-stratification of field measurements. In the 
213 second section we evaluate our ability to make inferences from our field data for subdomains

214 (Portland, Seattle, eastern Oregon and Washington) and for subgroups (diameter classes and

215 species). In the third section we evaluate i-Tree Eco software to support inferences from these

216 data.

\section{Post-Stratification}

218 We use subplot-level data to assess the correspondence between field measured land use and

219 NLCD land use classes to obtain a measure of the accuracy of the NLCD data. The other

220 analyses in this section were based upon plot-level classifications. In both analyses, we followed

221 an estimation strategy to classify the plot by using the NLCD classification observed at subplot

222 center (land use recorded at the center of subplot 1 represented the plot-level observation).

223 Associations between NLCD and FIA Land Use classes

224 NLCD accuracy was evaluated by examining the association between NLCD classes and land

225 use classes on subplots. Subplots are used here instead of plots, because plots may straddle

226 multiple land use classes, where combining subplots adds additional noise. A contingency table

227 for NLCD and land use classes with more than 10 observations (marginal totals) was prepared

228 (not shown) and used to compute association statistics between NLCD classes and field

229 measured land use classes. A simplification strategy was used to group NLCD and FIA land use

230 classes into like land covers which generally translate to levels of forest cover (bare, mixed bare

231 and forest, forest). The simplification is based on our objective to explain variability in the

232 spatial distribution of trees. In Table 2 we observe that all four measures of association indicate a

233 non-zero (and highly significant) association for both the grouped and un-grouped classes, but 
234 the strength of the association (variation described for numbers of subplots by land use class) is

235 weak.

236

237

Table 2

239 Post-stratification potential for land use classes

240 To assess the potential for post-stratification by land use classification we examined the amount

241 of separation in response variables when grouped by field-measured FIA land use classes. If this

242 classification scheme cannot aid in separation, it may suggest that land use is not a helpful

243 variable for post-stratification of urban inventory plots in Oregon and Washington, even under

244 ideal circumstances. In Fig. 3 we can see while the confidence intervals for BA and TPH (DBH

$245>12.7 \mathrm{~cm}$ ) on accessible forest land do not overlap the other land uses (bare and mixed), the

246 individual TPH and BA values on timberland do not appear to indicate that post-stratifying on

247 timberland would, on average, appreciably reduce the variances within post-strata.

Figure 3. Scatter plots and 95\% CIs for means of BA and TPH by FIA land use for land use classes with more than 10 field plots.

250 Homogeneity of responses by NLCD classes

251 Boxplots of TPH versus NLCD classes in Fig. 4 indicate that un-grouped NLCD classes

252 provided poor separation of TPH and BA into homogeneous groups. 
Figure 4. Boxplot of TPH and BA by NLCD class on plots.

255 Our findings in the previous section for FIA land use are reflected here as well for NLCD

256 classes. Fig. 5 shows mean estimates and 95\% confidence intervals for TPH and BA estimates

257 after grouping NLCD classes. For BA, the confidence interval for the mean in the forest class

258 does not overlap the other groups, but the spread of points does not indicate that the standard

259 deviation within groups is reduced by grouping observations. Two of the elevated TPH and BA

260 values for bare land reflect misclassification of timberland (FIA) as pasture (NLCD) and a

261 Christmas tree plantation (FIA) as cultivated crops (NLCD).

Figure 5. Scatter plots and 95\% CIs for means of BA and TPH by grouped NLCD classes with more than 10 field plots.

264 If we concentrate on trees larger than $12.7 \mathrm{~cm}$ (Fig. 6) we still do not see reasonable separation

265 between groups, although without the smallest trees the confidence interval for TPH in forest

266 lands does not overlap the two alternate grouped NLCD classes.

Figure 6. Scatter plots and 95\% CIs for means of BA and TPH by grouped

NLCD classes for classes with more than 10 field plots and for trees larger than $12.7 \mathrm{~cm} \mathrm{DBH}$.

\section{Effect of post-stratification on estimation precision}

269 The results in Table 3 agree with our graphical findings from previous sections. When estimating

270 population means for TPH and BA, post-stratification by NLCD classes does not appear to

Table 3 
271 increase our estimation precision. Standard errors for mean estimates following post-

272 stratification are not appreciably different from standard errors without post-stratification.

273 Standard errors for mean estimates following direct post-stratification with NLCD classes

274 (instead of overall estimates and precisions) are not provided because many of the classes had 275 small numbers of observations (1 to 20).

\section{Estimates for subdomains (subregions) and subgroups}

277 Under ideal circumstances we would leverage post-strata to increase the precision of our 278 estimates, but due to our finding that NCLD was not helpful for post-stratification, estimates for

279 this section were computed without post-stratification. We initially look at whether the data 280 support inferences base variables (TPH and BA) for selected subdomains, and then examine 281 whether the data support inferences about subgroups and subgroups within subdomains.

\section{Estimation for subdomains}

283 Estimation in areal subdomains is equivalent to post-stratification by geographic boundaries. The

284 objective is primarily motivated by our desire to make inference about sub-units in the

285 population; there is no assumption that post-stratification on region will reduce the sampling

286 variation within sub-region. The variation by species in sub-regions decline in some instances,

287 however this is primarily the result of having smaller mean values, but in some instance results

288 from reduced variability due to a smaller geographic extent. The numbers of observations in each

289 post-strata are smaller than for the population, which will on average increase standard errors

290 (decrease estimation precision) for sub-regions relative to estimation for the entire data extent. 
291 The results in Table 4 represent estimates of TPH and BA for sub-regions of our area-of-interest.

292 These results suggest that at the state level we can make basic inferences regarding TPH and BA.

293 However, the magnitudes of standard errors for mean estimate with these data in subdomains

294 indicate that this estimation strategy (including sampling design) provide minimal support for

295 subdomain inferences. For example, the basal area in Seattle is $60 \%$ greater than in Portland, a

296 difference which is likely of practical significant if substantiated. However, when we test the

297 probability that this difference is statistically significant with a Welch's t-test (Welch, 1938), we

298 observe a p-value larger than 0.08 which indicates that we do not have the statistical power to

299 identify this size of difference between these cities.

Table 4

304 The precision of estimates by subgroups, for example TPH or BA by species and DBH, will 305 generally be lower relative to the mean than for the base variables. The greater percent variation 306 in subgroups is the result of having fewer trees per plot to use for estimation. This will, for 307 example, result in a greater number of plots with zero values in the subgroup, which increases 308 the size of the variance relative to the size of the mean.

309 Increased variability for subgroups is demonstrated in Tables 5 and 6 in which we observe that 310 percent standard errors for individual species and diameter classes all exceed the standard errors 
311 for the corresponding base variables and sub-regions in Table 4. The magnitudes of standard

312 errors for subgroups indicate that, as with the base variables, the data cannot be used to support

313 inferences for subgroups between sub-regions. However, the data do in some cases support

314 inferences about subgroups for the entire region and in larger sub-regions (Washington and

315 Seattle).

Table 5

Table 6

319 For the most populous species in Table 5, we are able to detect practically significant differences

320 between species. For example, the difference between TPH for Pseudotsuga menziesii (Mirb.)

321 Franco (Douglas-fir) and Thuja plicata Donn ex D. Don (western red cedar) for all of Oregon

322 and Washington is arguably of practical significance (a difference of 4 TPH or 140\% larger than

323 Thuja plicata), but we would argue that the difference between Pseudotsuga menziesii and Alnus

324 rubra Bong. (red alder) (1.2 TPH or $20 \%$ larger than Alnus rubra) is not practically significant.

325 A paired t-test for the difference between Pseudotsuga menziesii and Thuja plicata was

326 significant $(\mathrm{p}=0.007)$, while the difference between Pseudotsuga menziesii versus Alnus rubra

327 was not signification $(\mathrm{p}=0.50)$. The differences between Pseudotsuga menziesii and Thuja

328 plicata were also significant for Washington State (.009) and Seattle (.01), but not for any of the 329 other sub-regions. 
330 The results for $5 \mathrm{~cm}$ diameter-class subgroups are similar to those for species in that we are

331 unable to compare across regions, but the data support some useful inferences within all of

332 Oregon and Washington and within specific sub-regions. The standard errors for small trees

$333(2.54 \mathrm{~cm}$ to $12.5 \mathrm{~cm})$ are especially large, mostly due to the reduced sample areas for small

334 trees, 0.0013 ha microplot versus 0.067 ha subplot for larger trees. However, for all of Oregon

335 and Washington, Washington and Seattle, most of the $5 \mathrm{~cm}$ DBH classes up to $48 \mathrm{~cm}$ were

336 significantly different from their neighbors at least at the 0.05 level. We also examined $10 \mathrm{~cm}$

337 diameter classes (table not shown), for which comparisons between the 3 smallest bins were

338 pairwise significant in all sub-regions except for Eastern Oregon and Washington.

339 When we examined TPH by species and diameter in combination (Table 7) the standard errors

340 become quite large relative to the means. It quickly becomes clear that attempting further

341 subdivision of the data by sub-regions will not support this level of analyses.

342

\section{Table 7}

343

$344 \quad$ i-Tree Eco software

345 Data initialization

346 Currently i-Tree Eco software cannot easily accept the inventory data collected by urban FIA.

347 The FIA data were collected on data loggers using Midas software which has a specific data

348 structure. Currently the i-Tree team is working on import facilities for FIA data. There is already

349 an import facility which accepts external data that has been properly coded and placed in excel 
350 tables. Users can also build their own import tools to place the data directly into a MS Access

351 database in the format used by i-Tree Eco. We used this approach (programmatic insertion of

352 FIA data directly into an MS Access database) to place our FIA data in the i-Tree Eco data.

353 Since i-Tree Eco provides a diverse array of outputs, the input data reside in a complex

354 arrangement of 32 interconnected tables in an MS Access database. We found that a minimum of

35511 of these tables were necessary to operate i-Tree Eco. However, once end users build a system

356 to link their records with the i-Tree Eco database, the programmatic solution is easy to repeat.

357 Reporting

358 i-Tree Eco provides a rich set of tables, figures, and a detailed textual report to describe and

359 provide background for many of the estimates in tables and figures. A list of available estimates

360 (related to trees) from i-Tree Eco, referred to by the software as "resource structural analysis

361 reports", is provided in Table 8.

362

Table 8

363

364 i-Tree Eco additionally provides estimates of trees' influence on air quality, ecosystem services

365 provided by trees, and information on trees' susceptibility to diseases. These should all prove

366 useful in the various planning and reporting demands for an urban forestry office.

367 While i-Tree Eco outputs extensive reports derived from the data, the format of the output

368 summaries is fixed and we felt that the selected format of the outputs inhibited interpretation. For

369 example we were interested in seeing trees per hectare by species and diameter class, however 
370 the report provides the percent of trees by diameter class and standard errors in number of trees.

371 In order to obtain estimates in trees per hectare would require a number of calculations by the

372 user - who may not be familiar with forest biometrics calculations. This makes it difficult to use

373 the report to obtain trees per hectare by diameter class. This style of reporting (tree per hectare in

374 percent and SE in number of trees) is opposite of what we are familiar with; we believe it is more

375 common and more interpretable to provide actual trees per hectare values and provide SE values

376 in percent (although reporting SE values in number of trees is also fine). There is probably not a

377 need for a custom reports module to meet each a multitude of user's different needs, but the

378 software authors may wish to investigate how mainstream forest inventory software systems

379 format their outputs.

\section{Statistical inference}

381 One of the strengths of the i-Tree Eco systems is that it generates a report based upon estimates

382 from submitted data. Ideally we could levy these values to understand a city or cities. However, a

383 requirement for sound inferences from these data is that $\mathrm{SE}$ values are available for reported

384 values and correctly calculated. If the report estimates that we have a lot of trees of a given

385 species, we can use the information to plan our management, but only if the SE values are

386 sufficiently small to suggest that estimate is reliable. If, for example, the SE value is as large as

387 the estimate then we would have some difficulty relying on the estimate (this suggests we need a

388 larger sample to be able to make inference about the estimate). In some instances it is reasonable

389 to omit SE values if they are available elsewhere, e.g. when reporting in graphical form, however

390 this is not always the case in the i-Tree eco reports. For example, standard errors are not 
391 available for tables or graphs of tree density by land use - the user must compute these values

392 from the total numbers of trees and divide by the number of acres. While the missing SE values

393 can be calculated by the user from other tables, it would prove beneficial to associate standard

394 errors with every mean or total estimate. We can imagine many users would not know to

395 calculated trees per hectare, or calculate standard errors for trees per hectare before they can

396 make inferences from reported values.

397 Most tables do have standard errors, however there is no documentation for how standard errors

398 were calculated. In a number of instances we attempted to reproduce the standard error values in

399 the report and were unable to do so. An example computational errors in SE can be seen in the

400 provided sample project which is distributed with i-Tree Eco as the "plot-based sample inventory

401 project". When looking at species composition by diameter class, for example, there are a

402 number of instances where there are estimates of number of trees by diameter and species for

403 which the standard errors are listed as zero. This suggests that the software is excluding plots

404 which do not have the observed species, resulting in severe under-estimates of error. Inclusion of

405 zero values is important in the correct calculation of standard errors.

\section{Discussion}

\section{Post-Stratification}

408 Unfortunately we did not observe any improvement in estimation performance as a result of 409 post-stratification on NLCD land use class. This is perhaps not a surprising result as land use 410 classification schemes are not optimized to explain variability in tree size, density, and species.

411 This was demonstrated even for the ideal case in which we had actual on the ground 
412 measurements of land use. This preliminary examination of NLCD data served as our first

413 attempt to post-stratify the Oregon and Washington urban inventory data. However, alternate

414 classification data may provide more explanatory power - such as a vegetation-specific Landsat-

415 based classification scheme, census demographics data, individual cities' land cover maps, or

416 active remote sensing.

417 It is not clear how effectively another Landsat based classification system such as LANDFIRE

418 (Rollins, 2009) would work for post-stratification in urban areas, but a Landsat based

419 classification system aimed at tree size, density, and species instead of land use could potentially

420 improve results. With respect to the majority of our study area, another alternative would be the

421 use of lidar. Lidar is available extensively (but not comprehensively) over both Seattle and

422 Portland metro areas ("Puget Sound LIDAR Consortium Home," n.d., "Oregon Lidar

423 Consortium," n.d.). Although there would be concerns about the various ages of the datasets, it

424 should prove feasible to identify coarse structure classes with respect to tree size and cover.

425 These may enable estimation from this small sample size at a finer resolution for sub-regions and 426 subgroups.

427 Estimation for sub-regions and subgroups

428 Estimation for sub-regions and subgroups was feasible for selected cases, however, due to the 429 small sample size and lack of stratifying variables our ability to make inferences about sub430 regions was limited. Inferences about trees in Portland and Seattle are likely of great interest to 431 many, but these data essentially do not support looking beyond the base variables for these sub432 regions. This is unfortunate given that it is likely that TPH by diameter class and species is of 
433 greater relevance than TPH alone. As previously mentioned, improved stratifying variables may

434 help in this respect, increasing the resolution (sub-regions and subgroups) for which it is possible

435 to make inference. However, given the sampling intensity of these data, it is still unlikely that

436 even with better stratifying variables that inference can, for example, be made from these data

437 about TPH by diameter class and species in sub-regions.

$438 \quad$ i-Tree Eco

439 While limitations are bound to be present in a software tool as complex as i-Tree Eco, the

440 software has a lot of potential as a tool to facilitate management and inferences regarding the

441 condition of urban trees (Nowak et al., 2013). The diversity of figures, tables, and information

442 that i-Tree Eco provides should prove helpful in preparing inventory data for human

443 interpretation. Once the data were loaded into the i-Tree Eco database a complete report and

444 numerous tables were available in less than 24 hours (the data are processed "in the cloud"). In

445 contrast, having every urban inventory group attempt to replicate the output from scratch would

446 be tedious and redundant and the results may not be comparable between organizations.

447 While we were impressed by the simplicity and extensive reporting capacity of i-Tree Eco, we

448 cannot yet recommend the software without an update to include measures of error for all

449 estimated values, better descriptions of the estimation procedure and reported values, and

450 perhaps a revised strategy for reporting estimates and standard errors. Fortunately, the

451 development process for i-Tree Eco appears to be fairly responsive to external feedback, and we

452 do not envision these issues being problematic to remediate. I-Tree Eco does have an import

453 facility, which should enable most users to adopt the software regardless of their data collection 
454 system - although some programming is recommended to guarantee consistency in transferring 455 data into the excel sheets to be imported by i-Tree Eco.

456 The limited number of reported statistics regarding urban trees is likely a stronger deterrent for

457 users wishing to support management of urban trees from their inventory information. In

458 evaluating this software it would be important to examine the outputs and evaluate whether they

459 are sufficient to support the needs of the organization. This software will lend itself best to users

460 who have minimal need to interact with the inventory database and do not require custom

461 outputs, or who are more interested in estimates related to ecosystem services or air quality. In

462 many instances having a rigid measurement and reporting protocol may even prove to be an 463 advantage with respect to minimizing complexity and overhead.

\section{Conclusions}

465 Since the majority of people in the U.S. live in urban areas, and it is well known that trees play

466 an important role in human well-being, understanding how trees are distributed in the built

467 environment is critical to assessing how they affect human lives with respect to both their impact

468 on the quality of human life and to the economic costs and value associated with the resource. In

469 light of the impacts trees have on humans, the newly collected forest inventory data collected in

470 a consistent manner across Oregon and Washington urbanized areas are an important resource

471 for assessing the condition of the urban environment. However, it is also important to recognize

472 the uses for which these data can be reasonably applied, and those for which additional data are

473 likely necessary. Our analyses suggest that even without effective post-stratification, these data

474 still enable defensible inferences about the distributions of trees by species and diameter at the 
475 state level. Estimation at the sub-region scale is also supported on a very limited basis, but would

476 require supplementary inventory data or effective auxiliary information in most cases.

\section{$477 \quad$ Future research}

478 Our next objectives for these data are to assess whether use of alternative auxiliary data for post479 stratification will better enable us to make inferences about sub-regions and subgroups.

480 Incorporating data from inventories performed by individual cities is another option, but given 481 increased precision, the consistent sampling and measurement protocol provided by urban FIA 482 data would provide greater flexibility for compatible inferences within and between regions. We 483 are also interested in examining the new urban FIA plot design (single $14.6 \mathrm{~m}$ radius, 0.067 ha 484 area), and examining its efficiency properties in the urban environment, especially its interaction 485 with post-stratification. The FIA plot is likely to be very efficient when using estimators 486 appropriate for simple random samples, however with post-stratification it may be that the larger 487 plot footprint could reduce estimation precision following post-stratification if there are a large 488 number of plots which straddle multiple post-strata.

\section{Acknowledgements}

491 We would like to thank the Pacific Northwest Research Station of the USDA Forest Service, the 492 Oregon Department of Forestry, and Oregon State University College of Forestry for supporting 493 this research. Funding for the collection of the urban FIA data and for a portion of this research 494 came from an American Recovery and Reinvestment Act of 2009 grant (WFM-2619-01FHC). 
495 We would like to thank David Nowak for providing feedback on our manuscript, and for helping

496 us to use i-Tree Eco. We also want to acknowledge our anonymous reviewers in helping us

497 improve the manuscript. 


\section{References}

499 Akbari, H., 2002. Shade trees reduce building energy use and CO2 emissions from power plants. Environmental Pollution, 116, S119-S126.

Cramér, H. 1946. Mathematical methods of statistics. Available at: http://www.cabdirect.org/abstracts/19481602629.html. Accessed July 26, 2013.

Cumming, A.B., M.F. Galvin, R.J. Rabaglia, J.R. Cumming, and D.B. Twardus. 2001. Forest health monitoring protocol applied to roadside trees in Maryland. Journal of Arboriculture 27(3): 126-138.

Cumming, A.B. 2007. Urban forests of Wisconsin: pilot monitoring project, 2002. U.S. Department of Agriculture, Forest Service, Northeastern Area, State and Private Forestry.

Cumming, A.B., D.B. Twardus, and D.J. Nowak. 2008. Urban forest health monitoring: Large scale assessments in the United States. Arboriculture and Urban Forestry 34(6): 341346.

D’Orazio, M. 2012. StatMatch: statistical matching. $R$ package version 1(0). Available at: http://cran.r-project.org/package=StatMatch. Accessed July 26, 2013.

Donovan, G.H., and D.T. Butry. 2009. The value of shade: Estimating the effect of urban trees on summertime electricity use. Energy and Buildings 41:662-668.

Donovan, G.H., and J.P. Prestemon. 2012. The effect of trees on crime in Portland, Oregon.

Donovan, G.H., D.T. Butry, Y.L. Michael, J.P. Prestemon, A.M. Liebhold, D. Gatziolis, and M.Y. Mao. 2013. The relation between trees and human health: evidence from the spread of the emerald ash borer. American Journal of Preventative Medicine, 44: 139-145.

Dunham, P., D. Weyermann, and D. Azuma. 2001. A comparison of stratification effectiveness between the National Land Cover Data set and photointerpretation in western Oregon. In Proceedings of the 3d annual forest inventory and analysis symposium, , p. 17-19. Available at: http://stary.wl.sggw.pl/Members/misioo/pubs/2003/3AnnualFIASymposiumTraverseCity 2001-gtr_nc230.pdf\#page=69. Accessed August 8, 2013.

Dwyer, J.F., H.W. Schroeder, and P.H. Gobster. 1991. The significance of urban trees and forests: toward a deeper understanding of values. Journal of Arboriculture 17(10): 276284. 
Fry, J.A., G. Xian, S. Jin, J.A. Dewitz, C.G. Homer, L. Yang, C.A. Barnes, N.D. Herold, and J.D. Wickham. 2011. National Land Cover Database for the Coterminous United States. Photogrammetric Engineering and Remote Sensing 77(9): 859-864.

Goodman, L.A., and W.H. Kruskal. 1954. Measures of Association for Cross Classifications. Journal of the American Statistical Association 49(268): 732-764.

Kardan, O., Gozdyra, P., Misic B, Moola F, Palmer LJ, Paus T, et al. 2015. Neighborhood greenspace and health in a large urban center. Scientific Reports 5:11610.

Kinzig, A.P., P. Warren, C. Martin, D. Hope, and M. Katti. 2005. The effects of human socioeconomic status and cultural characteristics on urban patterns of biodiversity. Ecology and Society 10(1): 23.

Kuo, F.E., and W.C. Sullivan. 2001. Environment and Crime in the Inner City Does Vegetation Reduce Crime? Environment and Behavior 33(3): 343-367.

Lake, M., P. Marshall, M. Mielke, A.B. Cumming, and D. Twardus. 2006. National Forest HealtH MonitoriNg Program Monitoring Urban Forests in Indiana: Pilot Study 2002, Part 1: Analysis of Field Methods and Data Collection. (Part 1). Available at: http://www.treesearch.fs.fed.us/pubs/12461. Accessed October 7, 2013.

Liknes, G. C., M. D. Nelson, D. D. Gormanson, and M. Hansen. 2006. The utility of the cropland data layer for forest inventory and analysis. In Proceedings of the 8th Annual Forest Inventory and Analysis Symposium, Washington DC, USA, , p. 259-264. Available at: http://ddr.nal.usda.gov/handle/10113/42048. Accessed August 8, 2013.

Nowak, D., 1994. Air pollution removal by Chicago's urban forest. Chicago's urban forest ecosystem: results of the Chicago Urban Forest Climate Project, 63-81.

Nowak D.J., D.E. Crane, J.C. Stevens. 2006. Air pollution removal by urban trees and shrubs in the United States. Urban Forestry and Urban Greening 4:155-123.

Nowak, D.J., and J.F. Dwyer. 2007. Understanding the benefits and costs of urban forest ecosystems. In Urban and community forestry in the northeast, Springer, p. 25-46. Available at: http://link.springer.com/chapter/10.1007/978-1-4020-4289-8_2. Accessed October 1, 2013.

Nowak, D.J., A.B. Cumming, D. Twardus, R.E. Hoehn, C.M. Oswalt, and T.J. Brandeis. 2011. Urban forests of Tennessee, 2009. Notes. Available at: http://treesearch.fs.fed.us/pubs/40246. Accessed October 1, 2013.

Nowak, D.J., D.E. Crane, and P. McHale. 2013. i-Tree Eco. USFS. Available at: http://www.itreetools.org/eco/. Accessed August 7, 2015. 
Oregon Lidar Consortium. Available at: http://www.oregongeology.org/sub/projects/olc/default.htm. Accessed August 7, 2013.

Puget Sound LIDAR Consortium Home. Available at: http://pugetsoundlidar.org/. Accessed August 6, 2013.

R Development Core Team. 2010. R: A Language and Environment for Statistical Computing. Vienna, Austria. Available at: http://www.R-project.org. Accessed June 12, 2010.

Riemann, R. 2003. Pilot Inventory of FIA Plots Traditionally Called 'nonforest.' . Gen. Tech. Rep. NE-312. Newtown Square, PA: U.S. Department of Agriculture, Forest Service, Northeastern Research Station. Available at: http://www.fs.fed.us/ne/newtown_square/publications/technical_reports/pdfs/2003/ne_gtr 312.pdf. Accessed July 11, 2014.

Ripley, B. 2012. RODBC: ODBC Database Access (2011). R package version 1.3-3.

Rollins, M.G. 2009. LANDFIRE: a nationally consistent vegetation, wildland fire, and fuel assessment. International Journal of Wildland Fire 18(3): 235-249.

Sanders, R.A. 1986. Urban vegetation impacts on the hydrology of Dayton, Ohio. Urban Ecology 9(3): 361-376.

Tallent-Halsell, N.G. (ed.). 1994. Forest health monitoring 1994 field methods guide. EPA/620/R-94/027. U.S. Environmental Protection Agency, Washington, D.C.

Theil, H. 1967. Economics and information theory. North-Holland Amsterdam. Available at: http://cds.cern.ch/record/432570. Accessed August 6, 2013.

U.S. Department of Agriculture Forest Service, 1992. Forest service resource inventories: an overview. Washington, DC: U.S. Department of Agriculture Forest Service, Forest Inventory, Economics, and Recreation Research. 39 p. http://www.fia.fs.fed.us/library/historical-documentation/ [Accessed August 2015)

U.S. Department of Agriculture Forest Service. 2012. 1 Forest inventory and analysis national core field guide: field data collection procedures for phase 2 plots. Version 6.0. Volume 1. 6th ed. Arlington, VA: U.S. Department of Agriculture Forest Service, Forest Inventory and Analysis Program.

U.S. Department of Commerce, Bureau of the Census. 2011. Urban and rural classification. Available at: http://www.census.gov/geography.html. Accessed August 7, 2015.

Ulrich, R.S., R.F. Simons, B.D. Losito, E. Fiorito, M.A. Miles, and M. Zelson. 1991. Stress recovery during exposure to natural and urban environments. Journal of environmental psychology 11(3): 201-230. 
595 Welch, B.L. 1938. The Significance of the Difference Between Two Means When the Population 596 Variances Are Unequal. Biometrika 29(3-4): 350-362.

597 Westphal, L.M. 2003. Urban greening and social benefits: a study of empowerment outcomes. $598 \quad$ Journal of Arboriculture 29(3): 137-147.

599 Wolf, K.L. 2003. Public reponse to the urban forest in inner-city business districts. Journal of $600 \quad$ Arboriculture 29(3): 117-126.

601 


\section{Figure captions}

603 Figure 1 FIA plot design

604 Figure 2 Urban areas in Oregon and Washington by sub-regions

605 Figure 3. Scatter plots and 95\% CIs for means of BA and TPH by FIA land use for land use 606 classes with more than 10 field plots.

607 Figure 4. Boxplot of TPH and BA by NLCD class on plots.

608 Figure 5. Scatter plots and 95\% CIs for means of BA and TPH by grouped NLCD classes with 609 more than 10 field plots.

610 Figure 6. Scatter plots and 95\% CIs for means of BA and TPH by grouped NLCD classes for 611 classes with more than 10 field plots and for trees larger than $12.7 \mathrm{DBH}$. 


\section{Urban FIA Plot}

Subplot

radius $=7.3$ meter

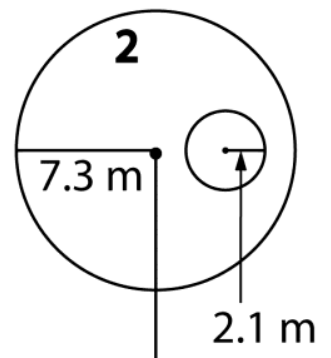

Microplot

radius $=2.1$ meter

located 90 degrees and

3.7 meters from subplot center

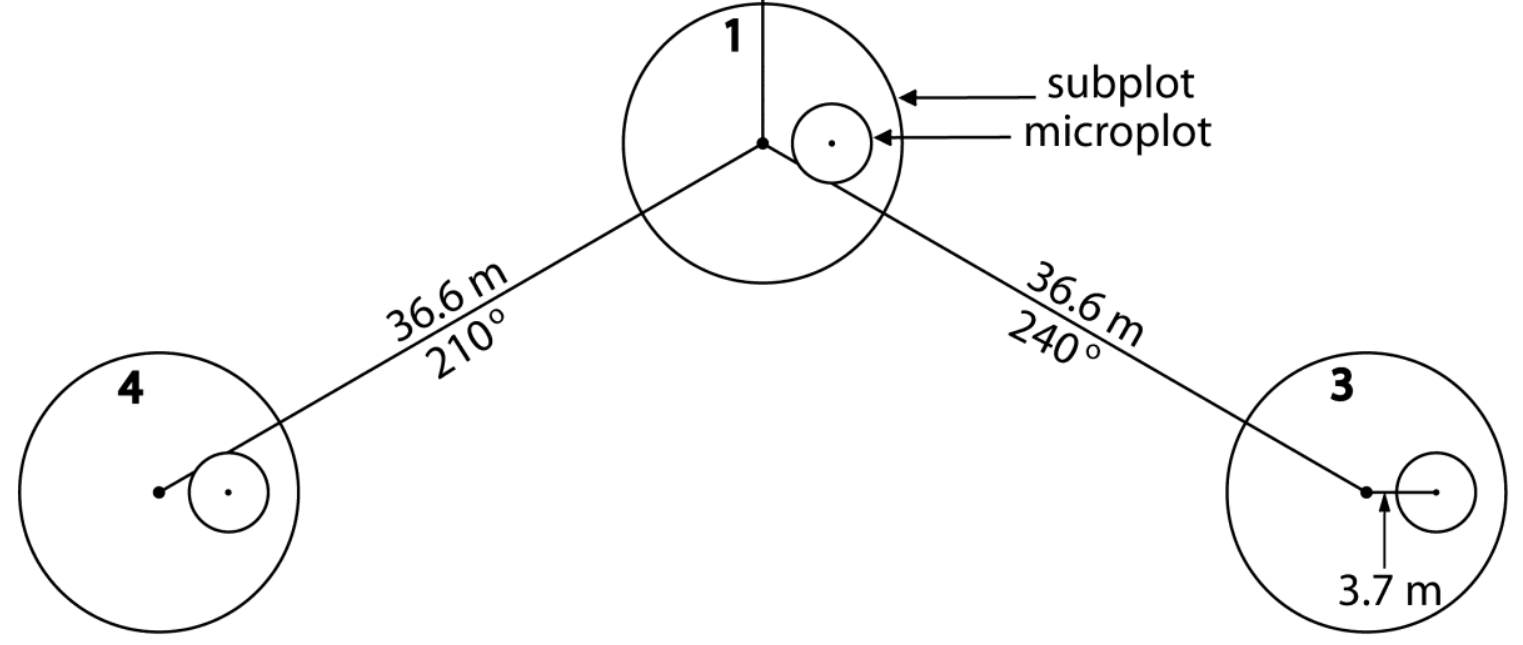

613

614 Figure 1. FIA plot design 


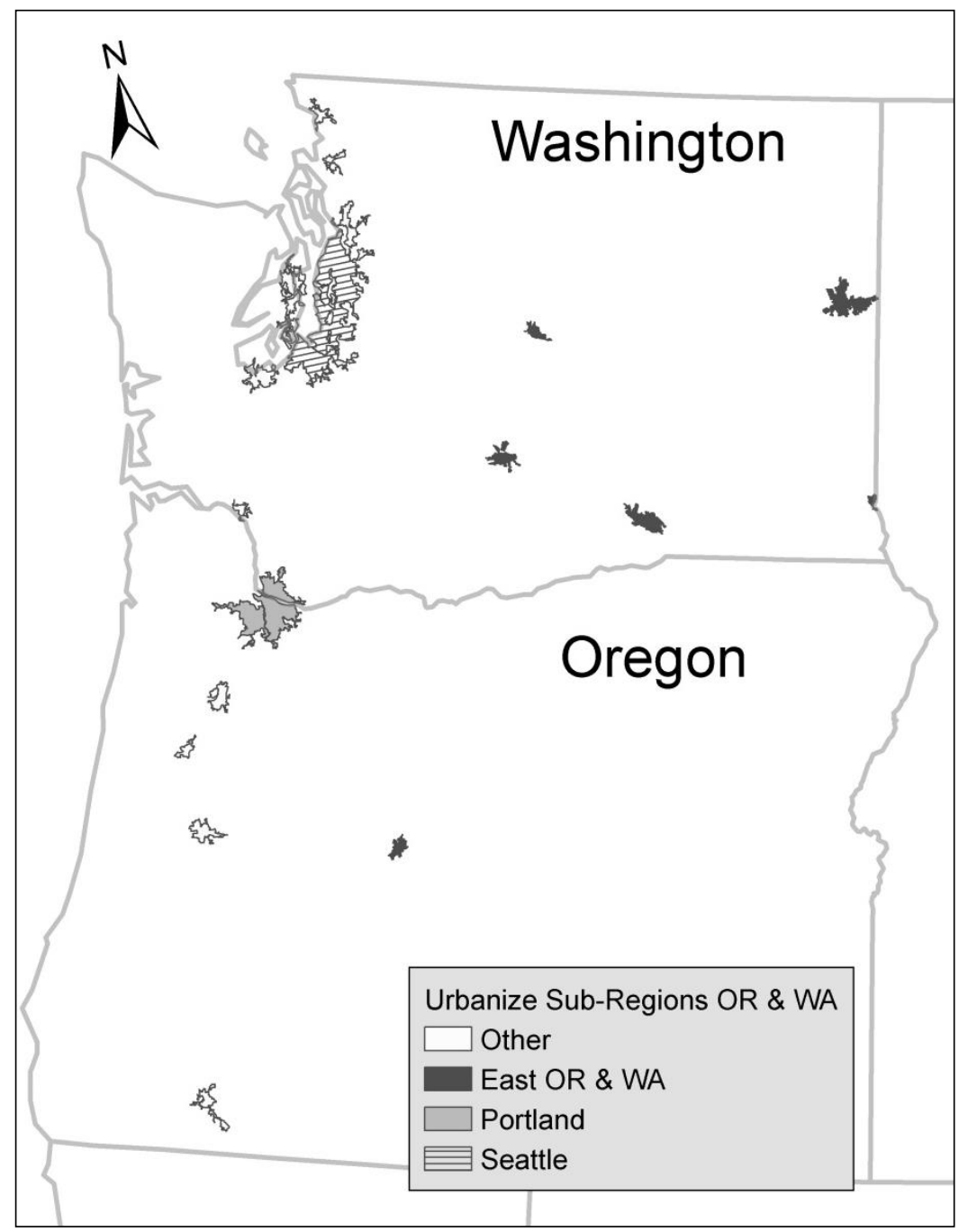

Figure 2. Urban areas in Oregon and Washington by sub-regions 

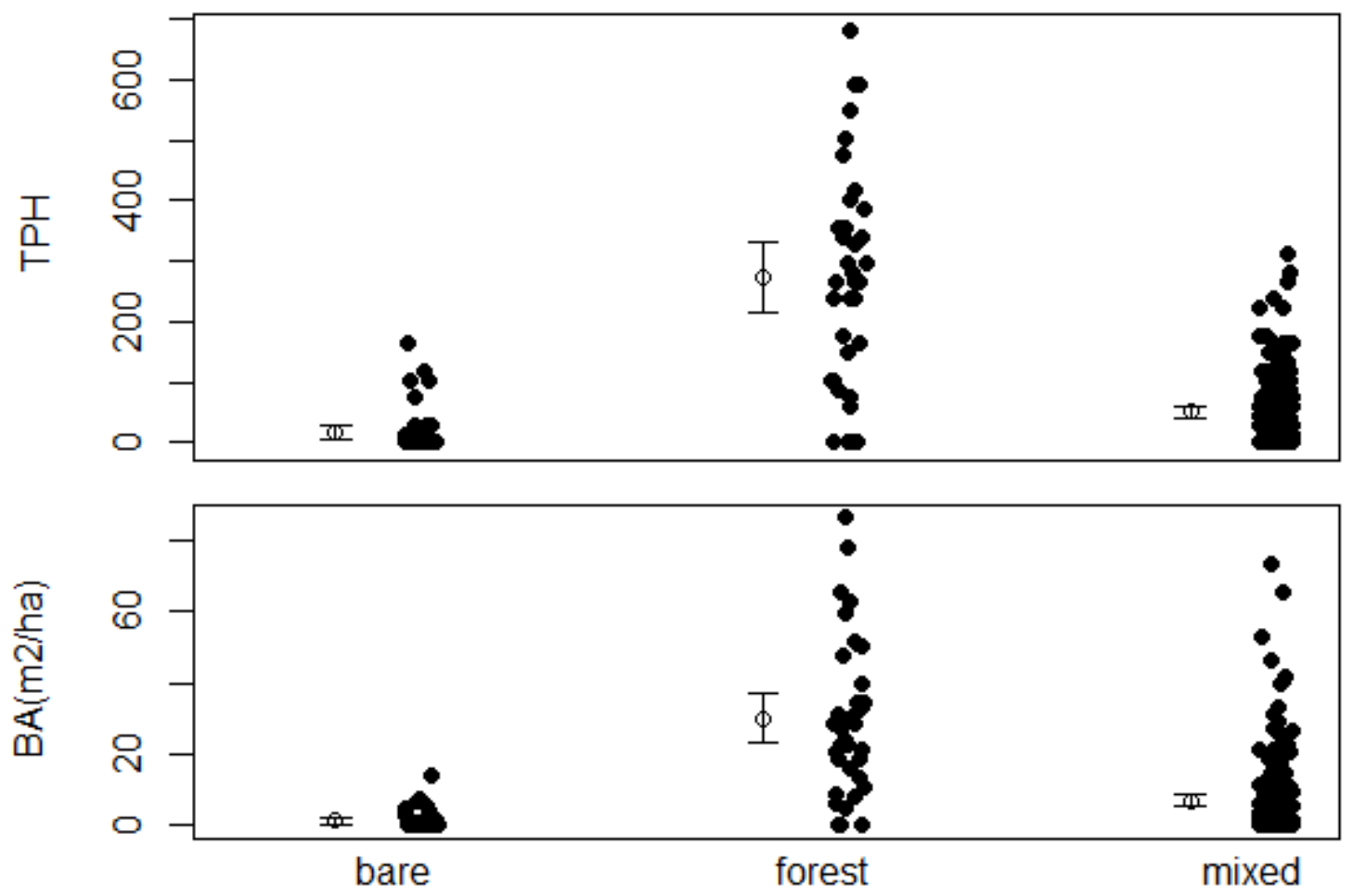

Figure 3. Scatter plots and $95 \%$ CIs for means of BA and TPH by FIA land use for land use classes with more than $\mathbf{1 0}$ field plots. 

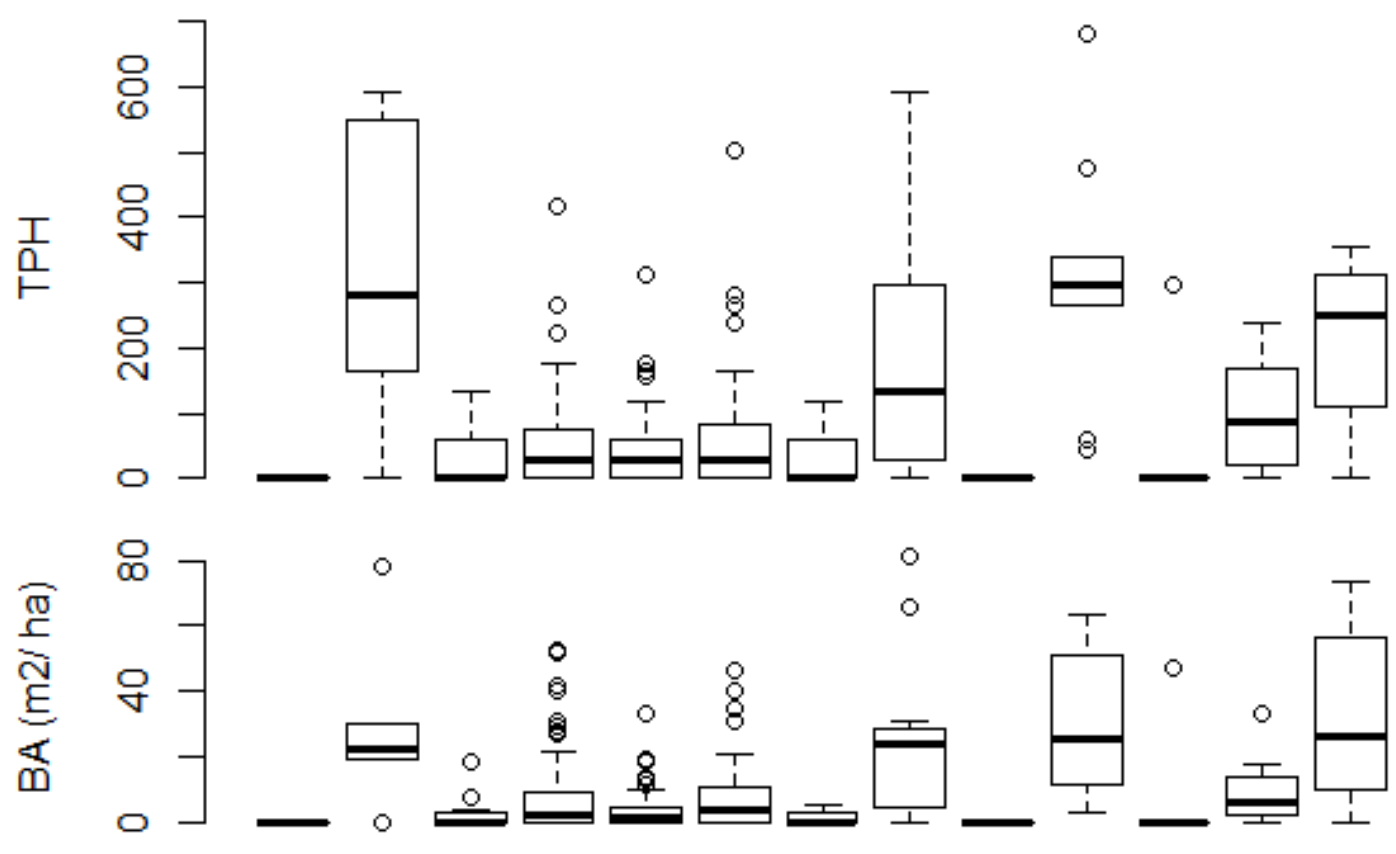

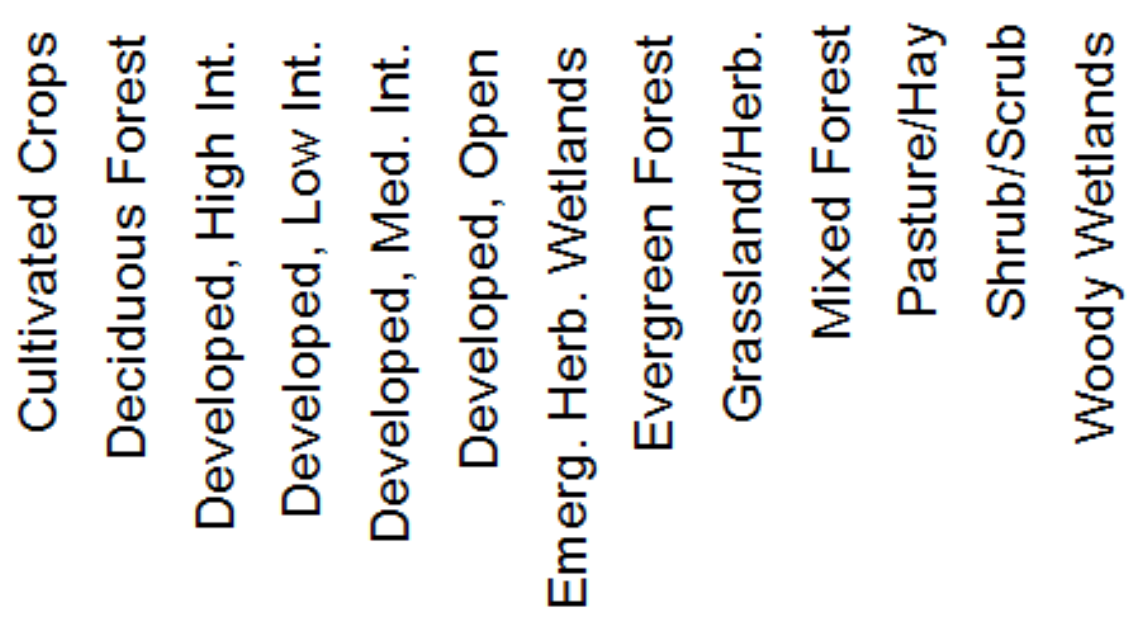

Figure 4. Boxplot of TPH and BA by NLCD class on plots. 

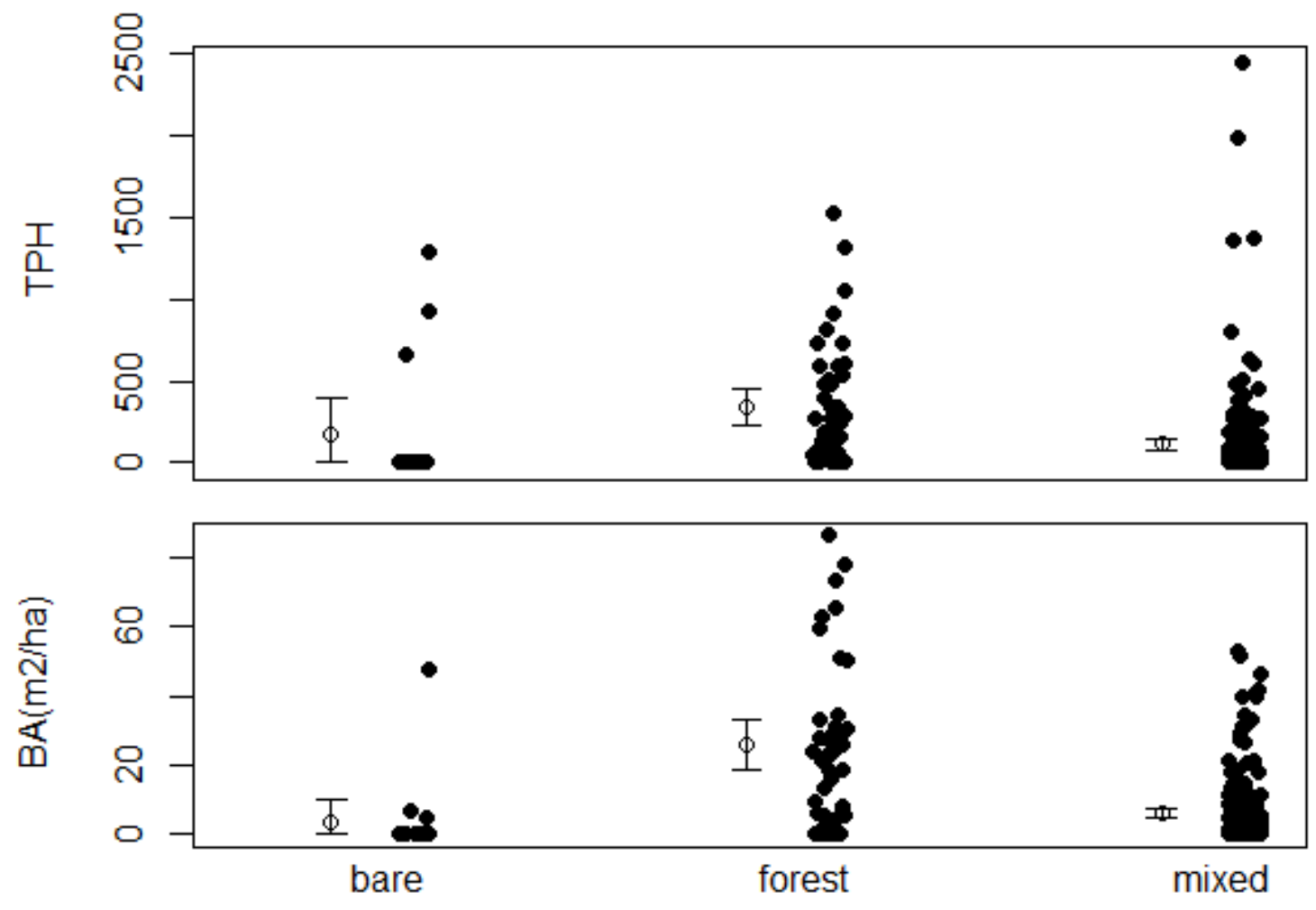

Figure 5. Scatter plots and 95\% CIs for means of BA and TPH by grouped NLCD classes with more than 10 field plots. 

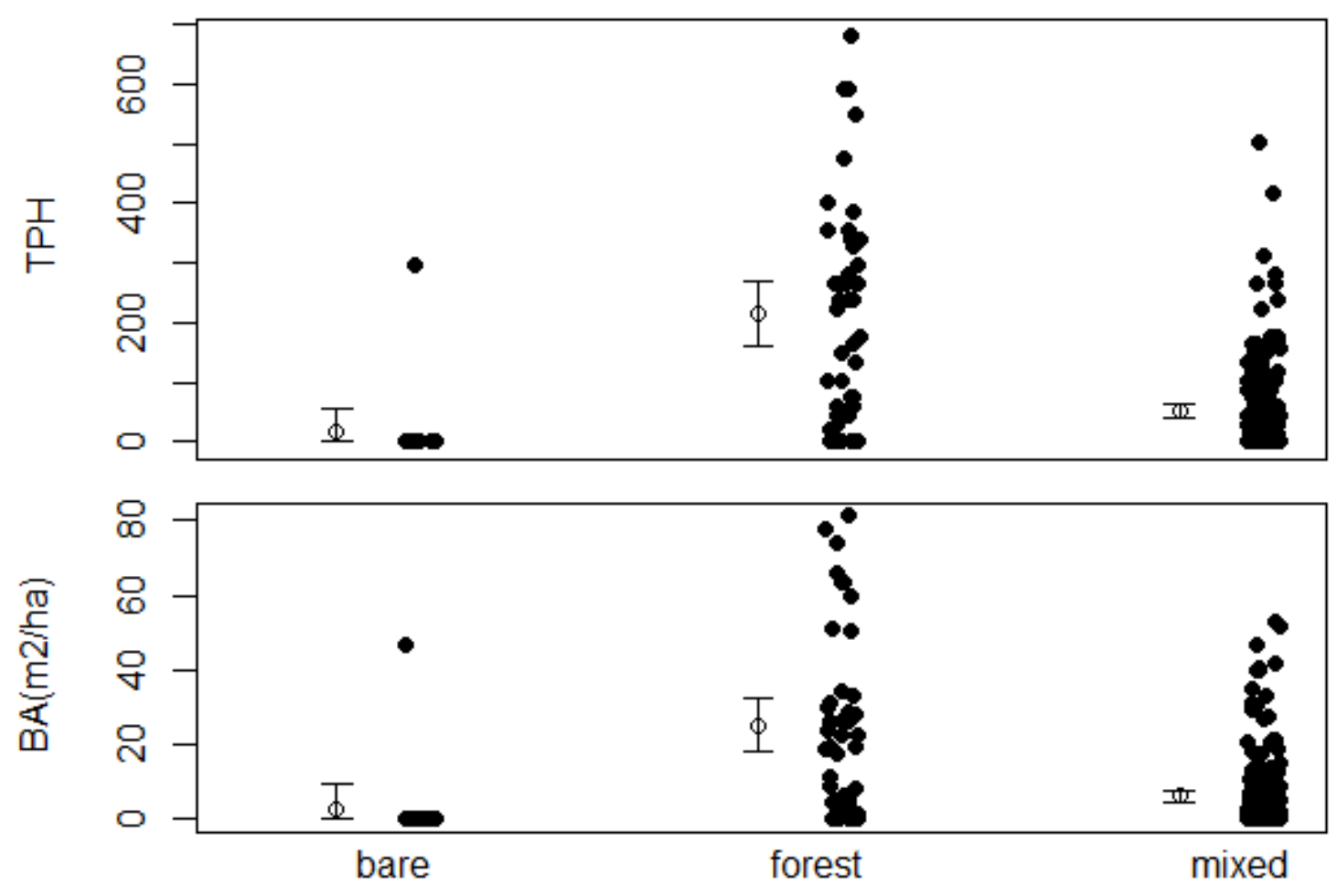

Figure 6. Scatter plots and 95\% CIs for means of BA and TPH by grouped NLCD classes for classes with more than 10 field plots and for trees larger than $12.7 \mathrm{~cm} \mathrm{DBH}$. 


\section{Tables}

Table 1. Summary statistics for selected field measured attributes.

Table 2. Statistical significance and measures of associations between NLCD and FIA land use classes on subplots when grouped and un-grouped.

Table 3. Mean estimates and standard errors (SE, percent of mean) for un-stratified (No), poststratified with NLCD (NLCD), and post-stratified with grouped NLCD observations (Grouped).

Table 4. Mean estimates and standard errors (percent of mean) for BA and TPH for the entire area and for sub-regions.

*Portland and Seattle refer to the Portland and Seattle standard metropolitan statistical areas respectively.

Table 5. TPH ( $>12.7 \mathrm{~cm}$ ) estimates and standard errors for the 11 most populous species (common names) for all of Oregon and Washington and for sub-regions.

*Portland and Seattle refer to the Portland and Seattle standard metropolitan statistical areas respectively.

Table 6. TPH estimates and standard errors for $5 \mathrm{~cm}$ diameter classes (DBCL) for all of Oregon and Washington and for sub-regions.

*Portland and Seattle refer to the Portland and Seattle standard metropolitan statistical areas respectively. 
Table 7. TPH by species and $5 \mathrm{~cm}$ diameter class (DBCL) for all of Oregon and Washington.

Table 8. Types of tree related estimates which can be acquired from i-Tree Eco. 
Table 1. Summary statistics for selected field measure attributes.

\begin{tabular}{lccccc}
\hline \hline & mean & med & sd & $\min$ & $\max$ \\
\cline { 2 - 6 } Tree DBH (cm, small*) & 6.7 & 6.3 & 2.7 & 2.5 & 12.4 \\
Tree DBH (cm) & 32.3 & 25.9 & 20.5 & 12.7 & 173.7 \\
Tree crown base ht. (m) & 7.5 & 5.0 & 6.8 & 0.0 & 38.3 \\
Tree crown density (\%) & 37.8 & 35.0 & 16.8 & 0.0 & 99.0 \\
Tree crown dieback (\%) & 3.9 & 5.0 & 4.9 & 0.0 & 99.0 \\
Tree crown width (m) & 7.2 & 6.7 & 3.2 & 0.3 & 34.6 \\
Tree height (m) & 18.6 & 17.1 & 9.6 & 1.5 & 58.8 \\
\hline \hline
\end{tabular}

*small tree measurements are from 110 live trees with dbh's less than $12.7 \mathrm{~cm}$. The remainder of the measurements used to prepare this table are from 1,273 live trees $12.7 \mathrm{~cm}$ in dbh and larger. 
1 Table 2. Statistical significance and measures of associations between NLCD and FIA land

2 use classes on subplots when grouped and un-grouped.

\begin{tabular}{ccc}
\hline \hline Assoc. Measure & Un-grouped & Grouped \\
\hline Cramer's V & 0.38 & 0.49 \\
Goodman - Kruskal lambda & 0.21 & 0.30 \\
Goodman - Kruskal tau & 0.18 & 0.28 \\
Theil's Uncertainty index & 0.24 & 0.27 \\
Chi-squared p-value & $<<0.001$ & $<0.001$ \\
\hline \hline
\end{tabular}

3

4 
5 Table 3. Mean estimates and standard errors (SE, percent of mean) for un-stratified (No), 6 post-stratified with NLCD (NLCD), and post-stratified with grouped NLCD observations

7 (Grouped).

\begin{tabular}{lcccc}
\hline \hline & \multicolumn{2}{c}{ All Trees } & \multicolumn{2}{c}{ Trees $<\mathbf{1 2 . 7} \mathbf{~ c m}$} \\
\hline & \multicolumn{2}{c}{ Mean } & SE & \multicolumn{2}{c}{ Mean } & SE \\
\cline { 2 - 5 } TPH - No & 157.2 & 19.7 & 78.4 & 7.4 \\
TPH - Grouped & 163.2 & 19.9 & 83.5 & 7.0 \\
BA - No & 9.5 & 1.0 & 9.1 & 1.0 \\
8 & 9.9 & 0.9 & 9.6 & 0.9 \\
\hline \hline
\end{tabular}


10 Table 4. Mean estimates and standard errors (percent of mean) for BA and TPH for the 11 entire area and for sub-regions.

\begin{tabular}{|c|c|c|c|c|c|c|}
\hline & \multicolumn{6}{|c|}{ Mean Estimate (Standard Error \%) } \\
\hline & $O R \& W A$ & OR & WA & East OR \& WA & Portland* & Seattle* \\
\hline BA & $9.5(10 \%)$ & $7.5(21 \%)$ & $10.1(12 \%)$ & $4.5(21 \%)$ & $7(25 \%)$ & $11(15 \%)$ \\
\hline $\mathrm{BA}(>12.7 \mathrm{~cm})$ & $9.1(11 \%)$ & $7.3(22 \%)$ & $9.8(12 \%)$ & $4(22 \%)$ & $6.6(27 \%)$ & $10.9(15 \%)$ \\
\hline TPH & $157.2(13 \%)$ & $154.7(25 \%)$ & $158.1(14 \%)$ & $167.5(33 \%)$ & $204.3(31 \%)$ & $134.9(16 \%)$ \\
\hline TPH $(>12.7 \mathrm{~cm})$ & $78.4(9 \%)$ & $59.7(19 \%)$ & $85(11 \%)$ & $47.3(25 \%)$ & $56.2(20 \%)$ & $96.8(14 \%)$ \\
\hline No. obs. & 257 & 67 & 190 & 37 & 65 & 102 \\
\hline
\end{tabular}

13 *Portland and Seattle refer to the Portland and Seattle standard metropolitan statistical 14 areas respectively. 
16 Table 5. TPH ( > $12.7 \mathrm{~cm}$ ) estimates and standard errors for the 11 most populous species

17 (common names) for all of Oregon and Washington and for sub-regions.

\begin{tabular}{|c|c|c|c|c|c|c|}
\hline \multirow[b]{2}{*}{ species com. } & \multicolumn{6}{|c|}{ TPH >12.7 cm Mean Estimate (Standard Error \%) } \\
\hline & OR\&WA & OR & $\bar{W} \bar{A}$ & East OR\&WA & Portland* & Seattle* \\
\hline Douglas-fir & $16.9(20)$ & $10.6(69)$ & $19.1(20)$ & $1.2(55)$ & $5.3(61)$ & $23.7(26)$ \\
\hline red alder & $13.7(25)$ & $5.3(69)$ & $16.7(27)$ & $0.4(99)$ & $9.8(56)$ & $19.6(31)$ \\
\hline bigleaf maple & $8.6(34)$ & $2(63)$ & $10.9(36)$ & $0.8(69)$ & $6.2(63)$ & $15.7(43)$ \\
\hline western redcedar & $7(23)$ & $3.8(47)$ & $8.1(26)$ & $0.8(99)$ & $4.2(50)$ & $6.7(36)$ \\
\hline ponderosa pine & $3.5(43)$ & $4.1(58)$ & $3.3(57)$ & $23.4(42)$ & 0 (NA) & 0 (NA) \\
\hline sweet cherry & $2.4(26)$ & $0.6(99)$ & $3(27)$ & $1.9(70)$ & $1.1(70)$ & $3.3(36)$ \\
\hline black cottonwood & $1.9(34)$ & $0.7(74)$ & $2.3(36)$ & $4.4(59)$ & $0.8(73)$ & $2.2(51)$ \\
\hline Pacific madrone & $1.6(61)$ & 0 (NA) & $2.2(61)$ & 0 (NA) & 0 (NA) & $3.5(70)$ \\
\hline western hemlock & $1.4(31)$ & 0 (NA) & $2(30)$ & 0 (NA) & 0 (NA) & $1.9(39)$ \\
\hline paper birch & $1(48)$ & $1.3(70)$ & $0.9(63)$ & $0(N A)$ & $1.7(70)$ & $0.7(59)$ \\
\hline Oregon white oak & $1(56)$ & $2.9(66)$ & $0.3(100)$ & 0 (NA) & $1.1(78)$ & $0.6(100)$ \\
\hline
\end{tabular}

18

19

20

21

*Portland and Seattle refer to the Portland and Seattle standard metropolitan statistical areas respectively. 
23 Table 6. TPH estimates and standard errors for $5 \mathrm{~cm}$ diameter classes (DBCL) for all of 24 Oregon and Washington and for sub-regions.

\begin{tabular}{|c|c|c|c|c|c|c|}
\hline \multirow[b]{2}{*}{ DBCL } & \multicolumn{6}{|c|}{ TPH Mean Estimate (Standard Error \%) } \\
\hline & OR \& WA & OR & WA & East OR\&WA & Portland* & Seattle* \\
\hline $2.5-7.4$ & $48.6(25)$ & $81.1(43)$ & $37.1(30)$ & $65.1(44)$ & $106.1(43)$ & $27.3(53)$ \\
\hline 7.5-12.4 & $29.2(32)$ & $11.7(46)$ & $35.4(35)$ & $50.1(50)$ & $39(72)$ & $11.2(45)$ \\
\hline $12.5-17.4$ & $18.9(12)$ & $15.3(21)$ & $20.1(14)$ & $11.2(23)$ & $17.1(25)$ & $22.5(18)$ \\
\hline 17.5-22.4 & $12.8(13)$ & $10.8(31)$ & $13.5(15)$ & $9.6(55)$ & $8.9(24)$ & $16.1(18)$ \\
\hline 22.5-27.4 & $11.8(13)$ & $9.4(33)$ & $12.7(14)$ & $8.2(28)$ & $8.2(34)$ & $13.9(14)$ \\
\hline 27.5-32.4 & $8.1(16)$ & $5.5(35)$ & $9(18)$ & $4.3(52)$ & $4.7(40)$ & $10.4(21)$ \\
\hline $32.5-37.4$ & $6(16)$ & $4.1(30)$ & $6.7(18)$ & 7) & $4.3(33)$ & $7.7(24)$ \\
\hline $37.5-42.4$ & 4.5 (17) & $2.2(35)$ & $5.3(19)$ & $2.4(44)$ & $2.2(41)$ & $6.2(25)$ \\
\hline $42.5-47.4$ & $3.2(18)$ & $2.4(38)$ & $3.5(20)$ & $1.6(59)$ & $1.7(45)$ & $3.3(27)$ \\
\hline 47.5-52.4 & 2.4 (19) & $1.8(42)$ & $2.6(21)$ & $1.6(59)$ & $1.7(45)$ & $3.7(25)$ \\
\hline 52.5-57.4 & $2(21)$ & $0.4(70)$ & $2.5(22)$ & $2.4(44)$ & $0.6(70)$ & $2.6(30)$ \\
\hline 57.5-62.4 & $1.6(22)$ & $1.8(49)$ & $1.6(24)$ & $1.2(55)$ & $1.7(61)$ & $1.9(30)$ \\
\hline $62.5-67.4$ & $1.2(31)$ & 0.2 (99) & $1.6(32)$ & 0.4 (99) & 0 (NA) & $1.9(42)$ \\
\hline $67.5-72.4$ & $1.2(27)$ & $1.3(51)$ & $1.2(31)$ & 0 (NA) & 1.4 (59) & 1.5 (39) \\
\hline 72.5-77.4 & $1(30)$ & $0.4(70)$ & $1.2(33)$ & 0.4 (99) & $0.3(99)$ & $1.2(42)$ \\
\hline $77.5-82.4$ & $0.6(43)$ & 0.2 (99) & $0.8(46)$ & 0.4 (99) & $0.3(99)$ & $1.2(55)$ \\
\hline $82.5-87.4$ & $0.5(33)$ & $0.4(70)$ & $0.5(37)$ & 0 (NA) & $0.3(99)$ & $0.7(44)$ \\
\hline $87.5-92.4$ & $0.4(42)$ & $0.4(70)$ & $0.4(53)$ & 0 (NA) & $0.3(99)$ & $0.4(74)$ \\
\hline
\end{tabular}

*Portland and Seattle refer to the Portland and Seattle standard metropolitan statistical areas respectively. 
TPH Mean Estimate (Standard Error \%)

\begin{tabular}{|c|c|c|c|c|c|c|c|c|c|}
\hline DBCL & 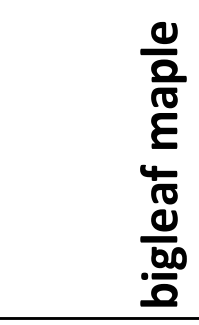 & 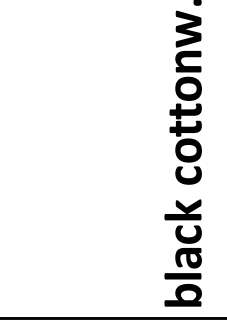 & 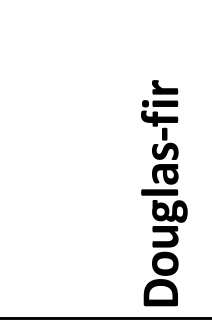 & 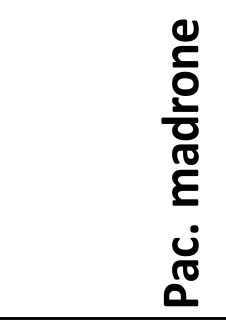 & $\begin{array}{l}\frac{5}{0} \\
\frac{.0}{0} \\
\frac{1}{0} \\
\frac{0}{0} \\
0\end{array}$ & 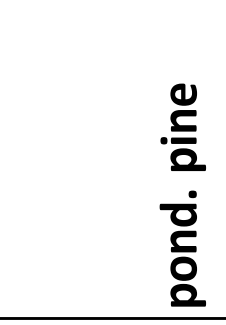 & $\begin{array}{l}\frac{\grave{d}}{0} \\
\frac{0}{\pi} \\
\frac{0}{0} \\
\underline{d}\end{array}$ & $\begin{array}{l}\frac{2}{2} \\
d \\
\frac{d}{U} \\
\dot{\varepsilon} \\
\frac{0}{0}\end{array}$ & 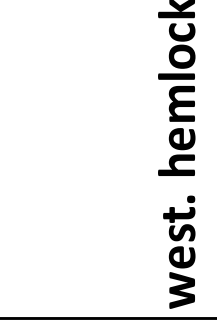 \\
\hline $0-4.9$ & - & 0.7 (100) & $5(87)$ & - & - & $2.2(100)$ & $7.9(73)$ & - & $1.4(100)$ \\
\hline $5-9.9$ & - & - & $5.2(61)$ & $0.1(100)$ & $0.8(93)$ & 0.7 (100) & $3.7(65)$ & - & 0.7 (100) \\
\hline $10-14.9$ & $1.4(40)$ & $0.6(37)$ & 2.2 (33) & $0.3(72)$ & $0.1(71)$ & $0.5(53)$ & $4(31)$ & $0.5(36)$ & 0.5 (39) \\
\hline $15-19.9$ & $1.1(34)$ & $0.2(50)$ & $2.4(33)$ & $0.3(53)$ & $0.1(100)$ & $0.9(76)$ & $2.5(31)$ & 0.9 (39) & $0.3(44)$ \\
\hline $20-24.9$ & $1.6(27)$ & $0.4(37)$ & $2.3(31)$ & $0.2(58)$ & $0.3(53)$ & $0.3(52)$ & $2.1(35)$ & $0.6(31)$ & $0.2(58)$ \\
\hline $25-29.9$ & $1.2(45)$ & $0.2(71)$ & $1.8(36)$ & $0.1(100)$ & $0.2(61)$ & $0.5(64)$ & $2(37)$ & $0.1(71)$ & $0.2(74)$ \\
\hline $30-34.9$ & $0.6(65)$ & $0.1(71)$ & $1(31)$ & $0.2(61)$ & $0.2(74)$ & $0.4(42)$ & $1.4(30)$ & $0.2(58)$ & - \\
\hline $35-39.9$ & $0.6(73)$ & $0.1(100)$ & $1.2(35)$ & $0.1(71)$ & $0.1(100)$ & $0.2(58)$ & 0.7 (39) & $0.1(100)$ & $0.1(71)$ \\
\hline $40-44.9$ & $0.4(55)$ & - & $1.3(25)$ & $0.2(100)$ & $0.1(100)$ & $0.2(74)$ & $0.4(51)$ & - & - \\
\hline $45-49.9$ & $0.3(44)$ & - & $0.8(40)$ & $0.1(100)$ & - & $0.2(74)$ & $0.3(53)$ & - & $0.1(71)$ \\
\hline $50-54.9$ & $0.3(47)$ & - & $0.4(46)$ & $0.1(100)$ & - & $0.3(53)$ & $0.1(100)$ & - & - \\
\hline 55-59.9 & $0.3(52)$ & $0.1(100)$ & $0.6(42)$ & - & - & $0.1(100)$ & - & - & - \\
\hline $60-64.9$ & $0.1(71)$ & $0.1(100)$ & $0.3(47)$ & - & - & $0.1(100)$ & $0.1(100)$ & - & - \\
\hline
\end{tabular}


31 Table 8. Types of tree related estimates which can be acquired from i-Tree Eco.

Number of trees by land use

Number of trees per unit area by land use

Species composition by DBH class and land use

Species composition by DBH class

Most important tree species

Species richness, Shannon/Wiener Diversity Index

Origin of trees by land use

Condition of trees by species

Condition of trees by land use

Condition of trees by $\mathrm{DBH}$ and land use

Leaf area of trees by land use

Leaf area of trees per unit area by land use

Leaf area and biomass of trees by DBH class and land use

Leaf area and biomass of trees by land use

Value of trees 